\title{
Perfil de Metilación de Genes Supresores de Tumores como Factor Pronóstico en Pacientes con Leucemia Mieloide Aguda
}

\author{
Promoter Methylation Profile in Tumor Suppressor Genes as \\ Prognosis Factor in Patients with Acute Myeloid Leukemia
}

"Soledad Reyes J.; "Priscilla Brebi M.; "Carmen Gloria Ili G.; *"Sergio Muñoz N.; *Angélica Melo A. \& **** Rafael Guerrero P.

REYES, J. S.; BREBI, M. P.; ILI, G. C.G.; MUÑOZ, N. S.; MELO, A. A. \& GUERRERO, P. R. Perfil de metilación de genes supresores de tumores como factor pronóstico en pacientes con leucemia mieloide aguda. Int. J. Morphol., 29(1):151-157, 2011.

RESUMEN: Existe creciente evidencia que apoya la presencia de un perfil de metilación específico para Leucemia Mieloide Aguda (LMA). La metilación de los islotes $\mathrm{CpG}$ en las regiones promotoras de los genes supresores de tumores es un importante mecanismo de control epigenético y participa en el silenciamiento transcripcional. Esto puede contribuir a un nuevo entendimiento de la biología de la enfermedad y vislumbrar nuevas oportunidades terapéuticas. Identificar el perfil de metilación de las áreas promotoras de un grupo de genes supresores de tumores; (p15, p16, ESR1, IGSF4, SOCS1, RARB y DAPK), y relacionar el estatus de metilación gen especifica o combinada con diferentes parámetros clínico patológicos. Se utilizaron muestras de sangre o médula ósea obtenidas al momento del diagnóstico de 33 pacientes con LMA, infantil y del adulto, recolectadas entre los años 1997 y 2008 en el Hospital Hernán Henríquez de Temuco. Se evaluó la presencia de hipermetilación mediante una Reacción de Polimerasa en Cadena Metilación Específica (MSP), previa modificación con bisulfito de sodio. La frecuencia de metilación de los pacientes estudiados fue de $88 \%, 27 \%, 27 \%, 21 \%$, 15\%, 3\% y 0\% para ESR1, RARb, IGSF4, p15, SOCS1, DAPK, y P16, respectivamente. La hipermetilación de P15 y RARb presentó una asociación significativa para una menor supervivencia en forma individual $(\mathrm{p}=0,03$ y $\mathrm{p}=0,02)$, y combinada ( $\mathrm{p}=0,002)$. No se encontraron diferencias significativas entre metilación y los otros parámetros clínicos analizados. Los pacientes con LMA presentan hipermetilación de la región promotora en algunos genes supresores de tumores, afectando negativamente la supervivencia. Esto pudiese eventualmente contribuir al establecimiento de un patrón de metilación determinado con utilidad clínica.

PALABRAS CLAVE: Leucemia mieloide aguda; Metilación; Genes supresores de tumores; Factor pronóstico.

\section{INTRODUCCIÓN}

La Leucemia Mieloide Aguda (LMA) es un trastorno de las células precursoras hematopoyéticas mieloides caracterizado por el bloqueo en la diferenciación, resultando en el crecimiento anormal de la población clonal de células neoplásicas o blastos. Esta alteración maligna lleva a la pérdida de la función normal, dando origen a sus manifestaciones clínicas: alteración en la oxigenación de los tejidos, trastornos en la coagulación y propensión a las infecciones resistentes a los tratamientos de rutina (Galmet et al., 2005).

La leucemia es el cáncer más comúnmente diagnosticado en pacientes pediátricos, con una incidencia global de 4.3 por cada 100.000 personas (Ries et al., 2006). La LMA afecta entre el $15 \%$ y el $20 \%$ de estos pacientes. El primer peak de incidencia ocurre dentro del primer año de vida y luego decrece hasta los 4 años, manteniéndose así hasta aproximadamente los 35 años (Aquino, 2002).

La LMA corresponde aproximadamente a un 25\% de todas las leucemias en los adultos en el mundo occidental, por lo tanto, es la forma más frecuente de leucemia en este grupo etáreo (Ries et al., Greenle et al., 2001). La LMA, es raramente diagnosticada antes de los 40 años, ya que, la incidencia incrementa progresivamente con la edad. Se ha

\footnotetext{
* Laboratorio de Patología Molecular, Departamento Anatomía Patológica, Facultad de Medicina, Universidad de La Frontera, Núcleo de Desarrollo Científico Tecnológico en Biorecursos (BIOREN), Temuco, Chile.

** Departamento de Salud Pública y del Centro de Excelencia CIGES, Facultad de Medicina, Universidad de La Frontera, Temuco, Chile.

*** The Johns Hopkins University, School of Medicine, Otolaryngology Department, Head and Neck Cancer Research Division, Baltimore, USA.
} 
estimado que en pacientes adultos menores de 65 años es de 1,8 por 100.000 personas, en cambio, la incidencia en mayores de 65 años presenta un aumento importante, estimándose una tasa de 17 por 100.000 personas (Ries et al.). Los pacientes recién diagnosticados tienen una edad promedio entre los 65 y 70 años en países desarrollados (Descher \& Lubbert, 2006).

En Chile se desconocen datos exactos, pero se calcula una prevalencia general aproximada de 2,6 por 100.000 individuos cada año (Hebel, 2009). Según datos publicados en la última década, la edad promedio es de 48 años para LMA adultos, M2 fue el tipo más frecuente y la mortalidad es del 90\% (Puga et al., 2000). En la LMA infantil, se presentan aproximadamente 25 casos nuevos por año, M2 fue también más frecuente, y la mortalidad a 5 años es de $63 \%$ (Quintana et al., 2005).

Ries et al. (2006), reportó en Estados Unidos , tasas de sobrevida a 5 años en aquellos pacientes menores de 55 años de $23 \%$, mientras que para aquellos mayores de 55 años de $11 \%$. Las tasas de sobrevida han mejorado en la última década dentro del primer grupo (de 9\% en 1980 a 35\% en 1990), pero no ha cambiado en el grupo de mayor edad, por lo que es este último el que representa el mayor desafío para alcanzar un mayor éxito terapéutico y mejorar la sobrevida en los pacientes con LMA.

A pesar de la relativa uniformidad en su presentación clínica, la LMA se caracteriza por una amplia variedad de anormalidades citogenéticas. La heterogeneidad molecular de la enfermedad se traduce en una respuesta variable a la quimioterapia estándar (Jabbour et al., 2006). Aunque para algunas alteraciones moleculares se ha demostrado una relación directa en la leucemogenesis, modelos animales han demostrado que LMA con translocaciones específicas y/o inversiones no son suficientes para causar leucemia (Gilliland et al., 2004).

Estudios moleculares han demostrado que alteraciones epigenéticas comparten un papel protagónico en el desarrollo de la LMA. La epigenética abarca una serie de alteraciones heredables en la expresión génica que no están causadas directamente por un cambio en la secuencia nucleotídica del ADN, como son las alteraciones de la estructura de la cromatina mediada por metilación de los residuos de citosina en los dinucleótidos $\mathrm{CpG}$, la modificación de histonas mediante acetilación o metilación y cambios en la estructuración jerárquica de la cromatina de orden mayor (Blum \& Marcucci, 2005). Al igual que las alteraciones genéticas, la ocurrencia de cambios epigenéticos es proclive a producir efectos funcionales. Es por ello que los cambios epigenéticos se han sugerido como biomarcadores de riesgo e indicadores de respuesta al tratamiento (Ross et al., 2005).

Los perfiles de metilación de los islotes $\mathrm{CpG}$ de los genes supresores de tumores varían según el tipo de tumor y son específicos para él (Esteller, 2007). Se considera que un gen tiene una alta frecuencia de metilación si esta es mayor a un $40 \%$, una frecuencia intermedia desde un $20 \%$ y una baja si es menor (Zavala et al., 2007). Se ha demostrado que varios genes supresores de tumores presentan hipermetilación en las áreas promotoras en la LMA. Esto es especialmente importante debido al creciente interés en el uso de los tratamientos con drogas demetiladoras. Aunque en sus inicios no siempre tuvieron como blanco la metilación, estas estrategias han demostrado eficacia en ensayos clínicos que han incluido pacientes con Síndrome Mieloproliferativo (Kantarjian et al., 2006) y LMA (Pinto \& Zagonel, 1993).

Dada la escasa información existente en nuestro país con respecto a cambios epigenéticos en pacientes con LMA se propuso identificar los perfiles de metilación de un grupo de genes supresores de tumores que se relacionan a las diferentes vías de la carcinogénesis en Leucemia Mieloide Aguda (p15, p16, ESR1, IGSF4, SOCS1, RARB y DAPK), y determinar su asociación con variables demográficas y de supervivencia.

\section{MATERIAL Y MÉTODO}

Muestras: Se seleccionaron 33 muestras de sangre o medula ósea de un grupo de pacientes con Leucemia Mieloide Aguda del Hospital Hernán Henríquez Aravena, cuyas muestras fueron enviadas para estudio de translocaciones al Laboratorio de Biología Molecular de la Facultad de Medicina de la Universidad de la Frontera, Temuco, Chile. Estas muestras fueron recolectadas entre los años 1997 y 2008. Los criterios de exclusión aplicados fueron: no contar con información suficiente para verificar el correcto diagnóstico y calcular la sobrevida, que las muestran fueron tomada en un momento diferente al diagnóstico, cambio posterior del diagnóstico inicial por considerar que los pacientes no correspondían a una LMA, sino a otro tipo de leucemia y finalmente, la no detección de la proteína constitucional Bglobina. Según el método descrito por Tu et al., el tamaño muestral es suficiente para determinar correlaciones entre genes, con un poder estimado de 0,78 (Tu et al., 2006).

Extracción de ADN: Se llevó a cabo con el Kit de extracción Puregene ${ }^{\circledR}$ Gentra System, el cual utiliza sangre total para la extracción de ADN. 
PCR beta globina: La integridad del ADN obtenido fue evaluada mediante la amplificación de un fragmento de 268pb del gen de beta-globina en condiciones de PCR descritas previamente (Herman et al., 1996).

Modificación de Bisulfito: Se realizó mediante el kit de conversión de bisulfito EpiTec de QIAGEN, según dos protocolos descritos por el fabricante. La eficiencia del Kit fue evaluada mediante PCR Beta actina con partidores específicos para muestras convertidas a bisulfito.

Metilación gen específico (MSP): Se determinó el estado de metilación de cada gen mediante MSP, utilizando pares de partidores dirigidos a sus regiones promotoras. Las secuencias de los partidores se obtuvieron de trabajos previamente publicados (Tabla I). Las condiciones de cada PCR fueron optimizadas tomando como referencia los trabajos citados. Como control positivo de la reacción se utilizó ADN 100\% metilado (Zymo Universal Methylated Human DNA Standard ${ }^{\mathrm{TM}}$ ) y como blanco mezcla de PCR sin ADN.

Análisis estadístico: Con el programa estadístico Stata 11, se realizaron tablas de contingencia para evaluar la frecuencia de metilación para cada uno de los genes, Se construyeron curvas de sobrevida de Kaplan-Meier, la comparación del tiempo de sobrevida entre metilados y no metilados se realizo mediante la prueba de logRank.

\section{RESULTADOS}

Características clínicas de la muestra: El número total de muestras analizadas fue similar para ambos géneros, representado por 17 hombres y 16 mujeres. La edad de los pacientes fluctuó entre 1 y 76 años con un promedio de 30 años. El 88\% correspondió a pacientes menores de 60 años. En relación a los exámenes complementarios realizados al momento del diagnóstico se obtuvo un promedio 35,557
Glóbulos Blancos por ul (GB). El promedio del recuento de plaquetas de diagnóstico fue de 46,185. La Hemoglobina presentó un promedio de 6.71. La mortalidad del grupo estudiado fue de un $84 \%$, mayor en los hombres que en las mujeres con un $93 \%$ y $76 \%$ respectivamente. No existieron deferencias significativas al comparar los valores por sexo. Las principales características clínicas de las muestras se describen en la Tabla II.

Frecuencia de Metilación génica en Leucemia Mieloide Aguda: La frecuencia de metilación de los pacientes estudiados fue de $88 \%, 27 \%, 27 \%, 21 \%, 15 \%, 3 \%$ y $0 \%$ para ESR1, RARb, IGSF4, p15, SOCS1, DAPK, y P16, respectivamente. La Figura 1 representa gráficamente la evaluación de la amplificación de los productos específicos de los MSP.
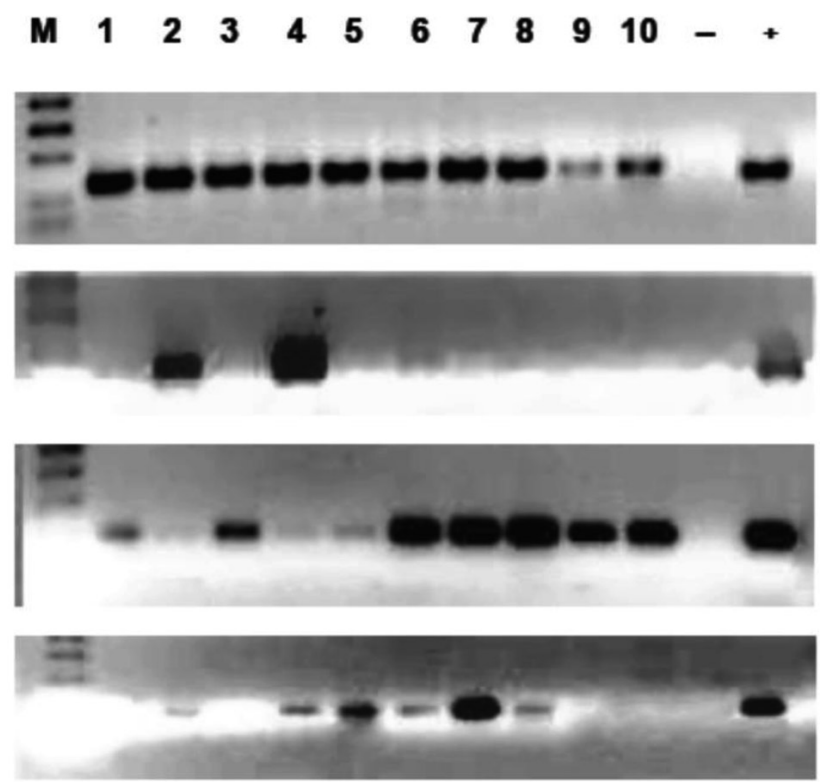

Fig. 1. Ejemplos representativos de el PCR control (b-actina) y los PCR específicos de metilación (MSP). M, marcador de peso molecular; 1-10, muestras; (-), control negativo; (+), control positivo, DNA 100\% Metilado-Modificado (Zymo Research).

Tabla I. Listado de Primers para PCR metilación gen especifica (MSP).

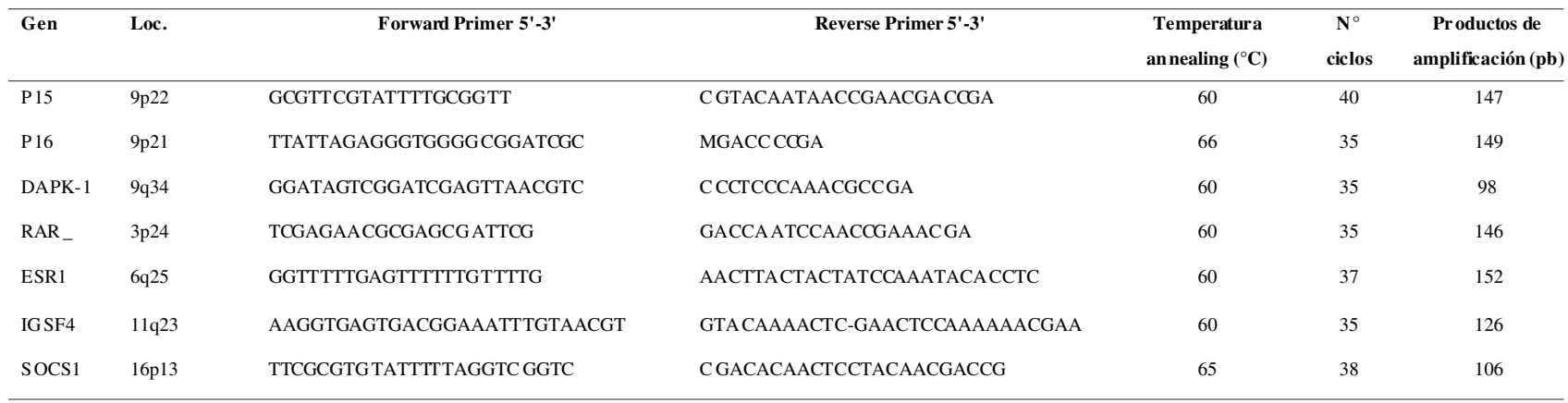


REYES, J. S.; BREBI, M. P.; ILI, G. C.G.; MUÑOZ, N. S.; MELO, A. A. \& GUERRERO, P. R. Perfil de metilación de genes supresores de tumores como factor pronóstico en pacientes con leucemia mieloide aguda. Int. J. Morphol., 29(1):151-157, 2011.

\begin{tabular}{lll}
\hline Características de los pacientes & & \\
\hline Total & $33(100 \%)$ & \\
Género & Masculino & $17(52 \%)$ \\
Edad & Femenino & $16(48 \%)$ \\
& $<60$ años & $29(88 \%)$ \\
& $>60$ años & $4(2 \%)$ \\
Subtipos de LMA & & Total $(\%)$ \\
& M0 & $0(0 \%)$ \\
& M1 & $3(9 \%)$ \\
& M2 & $7(21 \%)$ \\
& M3 & $5(15 \%)$ \\
& M4 & $9(27 \%)$ \\
& M5 & $2(6 \%)$ \\
& M6 & $0(0 \%)$ \\
Media de parámetros & M7 & $0(0 \%)$ \\
(rangos de presentación) & No disponibles & $7(21 \%)$ \\
& Glóbulos blancos & $35557(500-328000) \mathrm{gb} / \mathrm{mm}^{3}$ \\
& Plaquetas & $46185(1600-435000)$ \\
& Hemoglobina & $9(2,7-13,6) \mathrm{g} / \mathrm{dL}$ \\
\hline
\end{tabular}

Tabla II. Características clínicas de las muestras de pacientes con leucemia mieloide aguda.

M0: Leucemia mieloblástica aguda sin diferenciación localizada.; M1: Leucemia mieloblástica aguda sin maduración; M2: Leucemia mieloblástica aguda con maduración; M3. Leucemia promielocítica aguda (con translocación t $(15 ; 17))$; M4 Leucemia mielomonocítica aguda (LMMA); M5.Leucemia monocítica aguda (LMoA); M6.Eritroleucemia aguda; son precursoras de globos rojos; M7. Leucemia megacariocítica aguda.
Perfil de metilación: Para obtener los perfiles de metilación se realizó un análisis exploratorio de los datos para los siete genes estudiados estableciendo el número de genes metilados por muestra. Solo dos pacientes presentaron un $57 \%$ de los genes metilados (4/7 genes). Tres casos presentaron $3 / 7$ genes metilados correspondientes al $43 \%$, el resto varió entre $2 / 7$ y $1 / 7$ genes por muestra ( $28,6 \%$ y $14,3 \%$ respectivamente). Solo un paciente no presentó metilación del área promotora de ninguno de los genes estudiados.

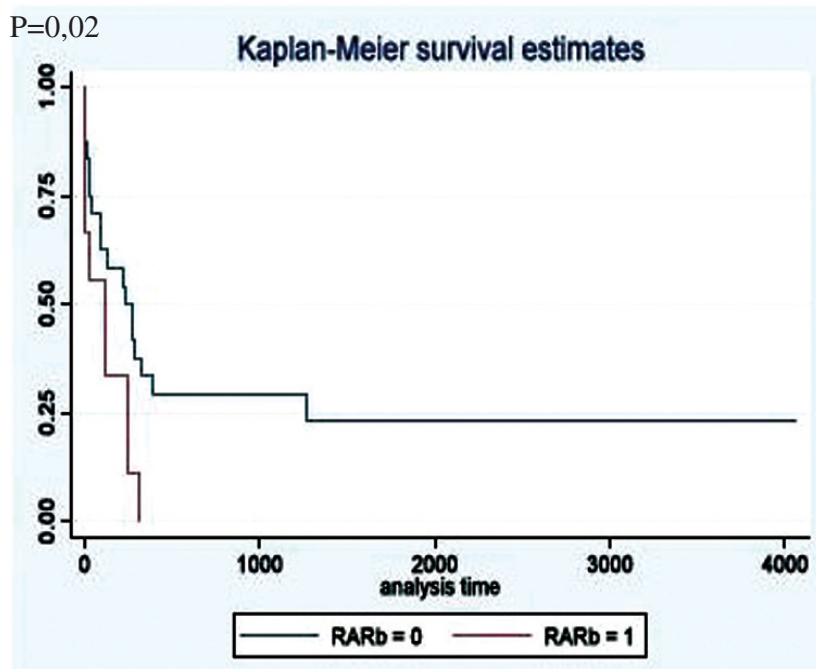

Fig. 2. Curvas de supervivencia en pacientes con leucemia mieloide aguda según estatus de metilación del gen p15, RARb y ambos. La linea azul, pacientes sin metilación. La linea roja, pacientes con metilación.
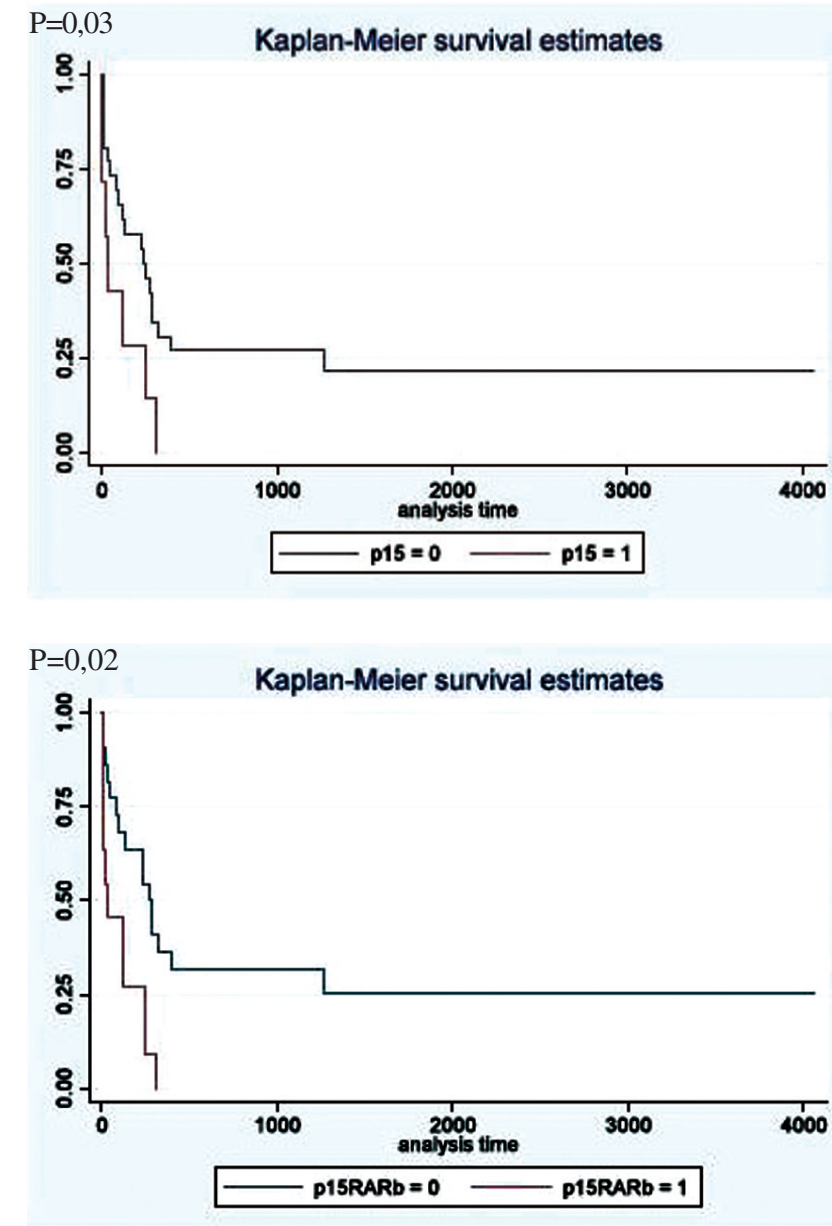
Secuenciación de bisulfito: Para evaluar si el fragmento amplificado correspondía al gen de interés, se realizó secuenciación de bisulfito. Los datos obtenidos fueron analizados mediante la plataforma Bioinformática Blast (http:/ /blast.ncbi.nlm.nih.gov/Blast.cgi). Los porcentajes de identidad observados indicaron que los fragmentos secuenciados corresponden a los genes de interés debido a que presentaron valores de Identidad superiores al $80 \%$. El gen P16 no fue secuenciado, debido a que no presentaba metilación en el área promotora en ninguna de las muestras analizadas.

Sobrevida de acuerdo a los resultados obtenidos los tiempos de sobrevida no difirieron significativamente para los genes ESR1, SOCS, IGSF4 y DAPk $(\mathrm{p}<0,05)$. Resultados estadísticamente significativos se obtuvieron para la hipermetilación de los genes p15 $(\mathrm{P}=0,03)$ y $\mathrm{RARb}$ $(\mathrm{P}=0,02)$. Esto deja de manifiesto que para estos genes existe diferencia en la sobrevida de pacientes que presentan el área promotora metilada con los que no presentan metilación. Al realizar el mismo análisis considerando la metilación del panel de los genes p15 y RARb, se observó una mayor sobrevida asociada al estado metilado $(\mathrm{P}=0,002)$ (Fig. 2).

\section{DISCUSIÓN}

La Leucemia Mieloide Aguda se presenta tanto en pacientes adultos como pediátricos, pero corresponde predominantemente a una enfermedad de la adultez tardía. El grupo de pacientes analizados presentó una edad promedio de 30 años, lo que es interpretado como un grupo relativamente joven comparado con el promedio de presentación de la enfermedad. Un estudio británico estimó que el $42 \%$ de sus pacientes eran mayores de 65 años y la edad promedio de presentación era de 65 años (Forman et al., 2003). Esto puede no ser homologable a nuestra población que es en promedio más joven. Es probable que esta diferencia se relacione también a un menor diagnóstico y que en nuestro país se produzca un mayor número de muertes por LMA en ancianos no diagnosticadas, debido a que los síntomas y signos se instalan mas lentamente y son menos evidentes que en pacientes jóvenes.

La edad de los pacientes que presentaron metilación en alguno de los genes estudiados levemente superiores al promedio. Esta diferencia fue más importante para el gen ESR1, el cual presentó un promedio de edad mayor en las muestras metiladas que en las que no se observó metilación -32 y 11 años respectivamente-. Aunque no constituye una diferencia estadísticamente significativa, sí existe una tendencia de asociación $(\mathrm{P}=0,08)$. Sin embargo, Hess y cola- boradores en el año 2008 registraron que la metilación del gen ESR1 se asociaba en forma significativa a una edad temprana de diagnóstico. Con similares resultados, existen diversos estudios anteriores que apoyan la teoría que los pacientes con LMA de mayor edad pueden tener una menor metilación de las áreas promotoras en algunos genes, lo que es especialmente observado en ESR1 (Hess et al.; Shimamoto et al., 2005). Estos resultados divergentes pueden ser consecuencia de la gran heterogeneidad de los pacientes con LMA y las diferencias en características clínicas y demográficas de los grupos estudiados.

La distribución por género en el grupo de estudio es muy similar: 52\% de hombres y $48 \%$ de mujeres. Esto concuerda con los datos que indican que la incidencia de LMA en adultos es levemente superior en hombres (Agrawal et al., 2007). El porcentaje de metilación para cada gen no presentó diferencias significativas según género.

Normalmente los glóbulos blancos (GB) presentan valores aproximados entre 4,500 y 10,000 unidades por microlitro. En los pacientes con leucemia estos valores pueden estar normales, disminuidos o aumentados al momento del diagnóstico, atribuyéndoles un valor pronóstico negativo cuando es mayor a 20,000 gb/mcL. El grupo de pacientes estudiados presentó un recuento de leucocitos promedio de $35,555 \mathrm{gb} / \mathrm{mcL}$, con cifras que estuvieron entre los 500$328.000 \mathrm{gb} / \mathrm{mcL}$. Los pacientes que presentaron el gen RARb hipermetilado, obtuvieron el promedio más alto de GB: $69,912 \mathrm{gb} / \mathrm{mcL}$; en contraste a los $22,114 \mathrm{gb} / \mathrm{mcL}$ de los no metilados. Esta diferencia presenta una tendencia de asociación, pero no alcanza a ser significativa $(\mathrm{p}=0,06)$.

Los datos obtenidos en este estudio, así como los anteriormente publicados que incluyen estudios de metilación en pacientes sanos en los genes estudiados (Hess et al.; Capello et al., 2008), muestran que las lesiones epigenéticas son abundantes y comunes en LMA, no asé en los pacientes sanos aumentando la posibilidad de que un número de lesiones oncogénicas sean de origen epigenético.

El análisis de la metilación de los genes calcitonina, receptor de estrógeno, E-cadherina, p15, p16, Rb, GSTP1 y HIC-1 en 20 pacientes con LMA, le permitió a Melki et al. definir la metilación simultánea en un 75\% de los casos con dos o más genes metilados (Melki et al., 1999). Toyota et al analizó 36 casos de LMA demostrando una alta frecuencia de metilación del gen ESR1, asociado con metilación de los genes MYOD1, SDC4, GPR37, y PITX2. La metilación de ESR1 también se ha relacionado a una mejor sobrevida. Por otro lado, Hess et al. recientemente analizó el estado de metilación de 25 genes supresores de tumor, utilizando MSMLPA (Methylation-specific multiplex ligation probe 
amplification); de ellos solo 3 resultaron metilados en forma significativa: ESR1, p15 e IGSF4.

Finalmente, este estudio identifica dos genes asociados a mal pronóstico antes descritos, p15 y RARb, y que usados en asociación constituyen un potente predictor de sobrevida, constituye también el primer acercamiento al estudio de la epigenética de la LMA en Chile.

Debido a que el ADN es estable y de rápida obten- ción de muestras clínicas, es altamente probable que una clasificación que incluya los perfiles epigenéticos servirá como un biomarcador de uso clínico usado para tomar decisiones en futuros ensayos clínicos.

Aunque la desregulación epigenética ha sido reconocida como una característica establecida del cáncer, el uso de la epigenómica para la expansión de nuestro entendimiento de la biología de esta enfermedad ha sido solo recientemente considerado en un contexto clínico.

REYES J. S.; BREBI M. P.; ILI G. C.G.; MUÑOZ N. S.; MELO A. A. \& GUERRERO P. R. Promoter methylation profile in tumor suppressor genes as prognosis factor in patient with acute myeloid leukemia. Int. J. Morphol., 29(1):151-157, 2011.

SUMMARY: There is growing evidence than acute myeloid leukemia presents a specific methylation profile. The Methylation of $\mathrm{CpG}$ islands within gene promoters is a major epigenetic transcriptional control mechanism and plays a critical role in the transcriptional silencing of tumor suppressor genes. This provides new insights into the biology of the disease and it may offer novel therapeutic opportunities. To identify the promoter methylation profile of tumor suppressor genes (p15, p16, ESR1, IGSF4, SOCS1, RARB y DAPK), and to relate the percentage of methylation with clinicopathological features, as age, gender, white cell count, disease classification and survival rates. Bone marrow and peripheral blood samples were collected at diagnosis from 33 patients with acute myeloid leukemia, infants and adult, between 1997 and 2008 from Hernán Henríquez Aravena Hospital, Temuco, Chile. Methylation in the promoter areas of each tumor suppressor gene was analyzed using the mehylation specific polymerase chain reaction (MSP) technique using sodium bisulfite modification. The frequency of hypermethylation among the patient samples was $88 \%, 27 \%, 27 \%, 21 \%, 15 \%, 3 \%$ and $0 \%$ for ESR1, RARb, IGSF4, p15, SOCS1, DAPK, and P16 for each one. Methylation was significantly associated with an inferior overall survival ( $\mathrm{p}=0.03$ and $\mathrm{p}=0.02$ ). When both genes are used, inferior survival is even more significant ( $\mathrm{p}=0.002$ ). There is no significant correlation between methylation and clinicopathological features.Patients with AML have hipermetilation at the promoter region of some tumor supressor genes, with a negative effect in the overall survival. This could eventually become part of establishing a characteristical methilation pattern with clinical utility.

KEY WORDS: Acute myeloid leukemia; Methylation; Tumor suppressor gene; Epigenetic.

\section{REFERENCIAS BIBLIOGRÁFICAS}

Agrawal, S.; Unterberg, M. et al. DNA methylation of tumor suppressor genes in clinical remission predicts the relapse risk in acute myeloid leukemia. Cancer Res., 67(3):1370-7, 2007.

Aquino, V. M. Acute myelogenous leukemia. Curr. Probl. Pediatr. Adolesc. Health Care, 32:50-8, 2002.

Blum, W. \& Marcucci, G. Targeting epigenetic changes in acute myeloid leukemia. Clin. Adv. Hematol. Oncol., 3:855-65, 2005.

Capello, D.; Deambrogi, C.; Rossi, D.; Lischetti, T.; Piranda, D.; Cerri, M.; Spina, V.; Rasi, S.; Gaidano, G. \& Lunghi, M. Epigenetic inactivation of suppressors of cytokine signalling in Philadelphia-negative chronic myeloproliferative disorders. Br. J. Haematol., 141(4):504-11, 2008
Deschler, B. \& Lubbert, M. Acute myeloid leukemia: epidemiology and etiology. Cancer, 107(9):2099-107. 2006.

Esteller. M. Epigenetic gene silencing in cancer: the DNA hypermethylome. Hum. Mol. Genet., 16:50-9, 2007.

Forman, D.; Stockton, D.; Møller, H.; Quinn, M.; Babb, P.; De Angelis, R. \& Micheli, A. Cancer prevalence in the UK: results from the EUROPREVAL study. Ann Oncol. 14:648-54, 2003.

Galm, O.; Wilop, S.; Lüders, C.; Jost, E.; Gehbauer, G.; Herman, J. G. \& Osieka, R. Clinical implications of aberrant DNA methylation patterns in acute myelogenous leukemia. Ann. Hematol., 84(Suppl. 1):3946, 2005.

Gilliland, D. G.; Jordan, C. T.; Jordan, C. T. \& Felix, C. A. 
REYES, J. S.; BREBI, M. P.; ILI, G. C.G.; MUÑOZ, N. S.; MELO, A. A. \& GUERRERO, P. R. Perfil de metilación de genes supresores de tumores como factor pronóstico en pacientes con leucemia mieloide aguda. Int. J. Morphol., 29(1):151-157, 2011.

The molecular basis of leukemia. Hematology Am. Soc. Hematol. Educ. Program., 80-97, 2004.

Greenlee RT, Hill-Harmon MB, et al. Cancer statistics, 2001. CA Cancer J. Clin., 51:15-36, 2001.

Gurney, J. G.; Severson, R. K.; Davis, S. \& Robison, L. L. Incidence of cancer in children in the United States. Sex, race-, and 1- year age-specific rates by histologic type. Cancer, 75:2186-95, 1995.

Hebel, E. Registros de Egresos Hospitalarios Servicio de Salud Araucanía Sur 2004-2009. Departamento de Estadística, Temuco, Chile, 2009.

Herman, J. G.; Graff, J. R.; Myöhänen, S.; Nelkin, B. D. \& Baylin, S. B. Methylation-specific PCR: a novel PCR assay for methylation status of $\mathrm{CpG}$ islands. Proc. Natl. Acad. Sci. USA, 93:9821-6, 1996.

Hess, C. J.; Errami, A.; Berkhof, J.; Denkers, F.; Ossenkoppele, G. J.; Nygren, A. O.; Schuurhuis, G. J. \& Waisfisz, Q. Concurrent methylation of promoters from tumor associated genes predicts outcome in acute myeloid leukemia. Leuk. Lymphoma, 49(6):1132-41, 2008.

Jabbour, E. J.; Estey, E.; Estey, E. \& Kantarjian, H. M. Adult acute myeloid leukemia. Mayo Clin. Proc., 81:247-60, 2006.

Kantarjian, H.; Issa, J. P.; Rosenfeld, C. S.; Bennett, J. M.; Albitar, M.; DiPersio, J.; Klimek, V.; Slack, J.; de Castro, C.; Ravandi, F.; Helmer, R. 3rd.; Shen, L.; Nimer, S. D.; Leavitt, R.; Raza, A. \& Saba, H. Decitabine improves patient outcomes in myelodysplastic syndromes: results of a phase III randomized study. Cancer, 106:1794-803, 2006.

Melki, J. R.; Vincent, P. C. \& Clark, S. J. Concurrent DNA hypermethylation of multiple genes in acute myeloid leukemia. Cancer Res., 59:3730-40, 1999.

Pinto, A. \& Zagonel, V. 5-Aza-2(-deoxycytidine (Decitabine) and 5-azacytidine in the treatment of acute myeloid leukemias and myelodysplastic syndromes: past, present and future trends. Leukemia, (Suppl. 1):5160, 1993.

Puga, L. B.; Cabrera, C. M. E.; Undurraga, S. M.; Etcheverry, B. R.; Vacarezza, Y. R.; Ducach, G. G. \& Toledo, G. H. Leucemia mieloide aguda del adulto: Resultados del Protocolo Nacional de Drogas
Antineoplásica. Hospital del Salvador 1990-1998. Rev. Méd. Chile, 128(11):1191-8, 2000.

Quintana, J.; Advis, P.; Becker, A.; Beresi, V.; Campbell, M.; Vinés, E. F.; García, H.; Salgado, C.; Vargas, L.; Rojas, J.; Obando, M.; Neira, L.; Páez, E. \& Zolezzi, P. Acute myelogenous leukemia in Chile PINDA protocols 87 and 92 results. Leukemia, 19(12):2143-6, 2005.

Ries, L. A. G.; Melbert, D.; Krapcho, M. et al., (ed). SEER cáncer Statistics Review, 1975-2004. Bethesda, MD: National cáncer Institute. Based on November 2005 SEER data submission, posted to the SEER website, 2006.

Ross, J. S.; Symmans, W. F.; Pusztai, L. \& Hortobagyi, G. N. Pharmacogenomics and clinical biomarkers in drug discovery and development. Am. J. Clin. Pathol., 124(Suppl):S29-41, 2005.

Tu, X. M.; Kowalski, J.; Crits-Christoph, P. \& Gallop, R. Power analyses for correlations from clustered study designs. Stat. Med., 25(15):2587-606, 2006.

Zavala, G. L.; Luengo, J. V.; Ossandón, F.; Riquelme, E.; Backhouse, C.; Palma, M.; Argandoña, J.; Cumsille, M. A. \& Corvalán, A. Identificación de asociaciones clínico-patológicas e hipermetilación de genes supresores de tumores en cáncer gástrico difuso a través de análisis de Hierarchical clustering. Rev. Méd. Chile, 135(1):17-25, 2007.

Dirección para correspondencia:

Dra. Soledad Reyes Jorquera

Facultad de Medicina

Universidad de La Frontera

Manuel Montt 112.

Código Postal 478-1176.

Temuco,

CHILE

Fono: 045-592148

Fax: 045-296530

E-mail: soledadreyes@ufro.cl

Recibido : 24-11-2010

Aceptado: 14-12-2010 